\title{
Evaluation of On- and Off-Line Bioluminescence Tomography System for Focal Irradiation Guidance
}

\author{
Bin Zhang, ${ }^{a}$ John W. Wong, ${ }^{a}$ Iulian I. Iordachita, ${ }^{b}$ Juvenal Reyes, ${ }^{a}$ Katriana Nugent, ${ }^{a}$ Phuoc T. Tran, ${ }^{a, c}$ Stephen W. \\ Tuttle, ${ }^{d}$ Constantinos Koumenis ${ }^{d}$ and Ken Kang-Hsin Wang ${ }^{a, 1}$ \\ ${ }^{a}$ Department of Radiation Oncology and Molecular Radiation Sciences, ${ }^{b}$ Laboratory for Computational Sensing and Robotics and ${ }^{c}$ Departments of \\ Oncology and Urology, Johns Hopkins University, Baltimore, Maryland; and "Department of Radiation Oncology, University of Pennsylvania, \\ Philadelphia, Pennsylvania
}

Zhang, B., Wong, J. W., Iordachita, I. I., Reyes, J., Nugent, K., Tran, P. T., Tuttle, S. W., Koumenis, C. and Wang, K. K-H. Evaluation of On- and Off-Line Bioluminescence Tomography System for Focal Irradiation Guidance. Radiat. Res. 186, 592601 (2016).

In response to the limitations of computed tomography (CT) and cone-beam CT (CBCT) in irradiation guidance, especially for soft-tissue targets without the use of contrast agents, our group developed a solution that implemented bioluminescence tomography (BLT) as the image-guidance modality for preclinical radiation research. However, adding such a system to existing small animal irradiators is no small task. A potential solution is to utilize an off-line BLT system in close proximity to the irradiator, with stable and effective animal transport between the two systems. In this study, we investigated the localization accuracy of an off-line BLT system when used for the small animal radiation research platform (SARRP) and compared the results with those of an on-line system. The CBCT was equipped on both the off-line BLT system and the SARRP, with a distance of $5 \mathrm{~m}$ between them. To evaluate the setup error during animal transport between the two systems, the mice underwent CBCT imaging on the SARRP and were then transported to the off-line system for a second CBCT imaging session. The normalized intensity difference of the two images and the corresponding histogram and correlation were computed to evaluate if the transport process perturbed animal positioning. Strong correlation (correlation coefficients $>0.95$ ) between the SARRP and the off-line mouse CBCT was observed. The offset of the implanted light source center can be maintained within $0.2 \mathrm{~mm}$ during transport. To compare the target localization accuracy using the on-line SARRP BLT and the off-line system, a selfilluminated bioluminescent source was implanted in the abdomen of anesthetized mice. In addition to the application for dose calculation, CBCT imaging was also employed to generate the mesh grid of the imaged mouse for BLT reconstruction. Two scenarios were devised and compared, which involved localization of the luminescence source

${ }^{1}$ Address for correspondence: Johns Hopkins University, Department of Radiation Oncology and Molecular Radiation Sciences, 401 N. Broadway, Suite 1440, Baltimore, MD 21231; e-mail: kwang27@ jhmi.edu. based on either: 1. on-line SARRP bioluminescence image and CBCT; or 2. off-line bioluminescence image and SARRP CBCT. The first scenario is assumed to have the least setup error, because no animal transport was involved. The second scenario examines if an off-line BLT system, with the mesh generated from the SARRP CBCT, can be used to guide SARRP irradiation when there is minimal target contrast in CBCT. Stability during animal transport between the two systems was maintained. The center of mass (CoM) of the light source reconstructed by the off-line BLT had an offset of $1.0 \pm 0.4 \mathrm{~mm}$ from the true CoM derived from the SARRP CBCT. These results are comparable to the offset of $1.0 \pm 0.2 \mathrm{~mm}$ using on-line BLT. With CBCT information provided by the SARRP and effective animal immobilization during transport, these findings support the utilization of an off-line BLT-guided system, in close proximity to the SARRP, for accurate soft-tissue target localization. In addition, a dedicated standalone BLT system for our partner site at the University of Pennsylvania was introduced in this study. ๑ 2016 by Radiation Research Society

\section{INTRODUCTION}

The development of novel methods for cancer treatment with radiation therapy relies on preclinical studies using small animal models. Small animal irradiation systems with computed tomography (CT)/cone-beam computed tomography (CBCT) guidance provide high-precision focal irradiation, which mimics human treatment in a clinical setting (1-5). Although CT/CBCT is invaluable for imageguided radiation therapy, the limited soft-tissue contrast prevents its application in targeting of orthotopic tumors, such as in liver, pancreas and prostate cancer models.

Bioluminescence imaging (BLI) is a noninvasive imaging modality with the favorable features of low cost, use of nonionizing radiation and high-contrast images (6-8). BLI offers an attractive solution for soft-tissue targeting, given that genetically engineered mouse models are becoming more widely available for oncologic and radiobiology research. In their published study, Baumann et al. (9) 
utilized two-dimensional (2D) BLI to localize orthotopic glioblastoma multiforme (GBM) tumors and facilitate radiation delivery using the small animal radiation research platform (SARRP). However, the common form of 2D bioluminescence image emitted from an animal surface is likely inadequate to guide radiation delivery. The surface light intensity is a function of the optical path from the source and is particularly confounded by the irregularly shaped animal. In our previously published work, we showed that a vertical beam directed at the highest bioluminescence intensity on the animal surface deviates from the center of mass (CoM) of an internal source by more than $3 \mathrm{~mm}(10)$. Including other reported sources of setup uncertainty (11), an undesirably large beam aperture $>15 \mathrm{~mm}$ in diameter, about half of the width of the mouse abdomen, would be required to ensure coverage of a small 3 $\mathrm{mm}$ diameter tumor. Bioluminescence tomography (BLT), based on the 2D bioluminescence image measured on the object surface, can reveal the three-dimensional (3D) distribution of the internal bioluminescent sources (1219). Recently, our group proposed an on-line 3D BLT system to guide SARRP irradiation (19).

Since their commercialized use five years ago $(1,4)$, over 70 small animal irradiation systems have been deployed in laboratories worldwide. Due to the potential limitations, such as space, cost and intricate methodology, it is challenging to add an on-line BLT system (19) to the existing SARRP for some institutes. In their recent published study, Tuli et. al. (20) evaluated the accuracy of using off-line BLI/BLT to guide irradiation by transporting an animal between a commercial bioluminescence system (IVIS ${ }^{\circledR}$ Spectrum; Caliper Life Sciences, Hopkinton, MA) and the SARRP. Because the BLI system is not in close proximity to the irradiator, at least $15 \mathrm{~min}$ were needed to transport the animal between the systems located at different facilities. In addition to the setup error introduced from the transport, the throughput was largely compromised.

A standalone BLT system in close proximity to the SARRP could be a potential solution to overcome these obstacles. To evaluate if the standalone system is adequate for bioluminescent target localization, we first evaluated the accuracy of animal positioning by transferring the mouse between a previously-described off-line CBCT/ BLT system (21) and the SARRP in close proximity. The mice CBCTs taken at both systems were analyzed and compared to assess the animal positioning before and after transport. We further compared the localization accuracy of bioluminescent targets between the on-line SARRP BLT (19) and the off-line BLT (21) systems. Our study results show that by utilizing the SARRP CBCT to generate the mesh information of the imaged object for the BLT reconstruction, a standalone BLT system without CBCT capability can provide reasonable target localization for the irradiator. These findings led us to develop a dedicated standalone BLT system for our partner site at the University of Pennsylvania, which was necessary to accommodate the space limitations in their SARRP room. An off-line BLT system in close proximity to the irradiator can be an alternative to on-line setup, if soft-tissue targeting is highly desired for an existing irradiator and longitudinal imaging studies without radiation treatment are needed.

\section{MATERIALS AND METHODS}

\section{On-Line SARRP BLT and Off-Line Systems}

The second-generation SARRP consists of a dual-focus X-ray source mounted on an isocentric gantry with $360^{\circ}$ rotation. The $\mathrm{X}$ ray source is used for both CBCT imaging ( $65 \mathrm{kVp}, 0.4 \mathrm{~mm}$ focus) and radiation delivery $(225 \mathrm{kVp}, 3 \mathrm{~mm}$ focus $)$. The animal positioning was controlled by a robotic stage. For CBCT imaging, the animal was rotated between the X-ray source and a complementary metal-oxide-semiconductor (CMOS) detector panel (200 $\mu \mathrm{m} /$ pixel, $20.5 \times 20.5 \mathrm{~cm}$; PerkinElmer ${ }^{\circledR}$, Waltham, MA) in horizontal position. In this work, $65 \mathrm{kVp}$ and $0.7 \mathrm{~mA}$ were used for CBCT imaging and the imaging volume was reconstructed as a $441 \times 441 \times 230$ matrix with a voxel size of $0.25 \times 0.25 \times 0.25$ $\mathrm{mm}^{3}$. To facilitate soft-tissue localization and targeting, our SARRP is equipped with an on-line BLT (19), as shown in Fig. 1A and C. The BLT system, including a camera-filter-mirror assembly and a light-tight enclosure, can be docked onto the SARRP for imageguide radiation therapy. Multispectral bioluminescence emitted from the imaging object was reflected to the CCD camera via a 3mirror system. This configuration is also applied to the off-line BLT system and shown in Fig. 1B. The mirror can rotate at $180^{\circ}$ and $90^{\circ}$ around the animal for the on- and off-line BLT system, respectively, to acquire multiple projection optical images. In this study, only single projection was used for both systems for BLT reconstruction. The CCD camera possesses a back-illuminated sensor $(2,048 \times$ 2,048 pixels, $27.6 \times 27.6 \mathrm{~mm}^{2}$; iKon-L 936, Andor Technology, Belfast, UK) with high quantum efficiency ( $>90 \%$ ) over $500-700$ $\mathrm{nm}$, which covers the spectral range of widely used bioluminescence imaging. It was operated at $-80^{\circ} \mathrm{C}$ to reduce the dark current and thermal noise during image acquisition. A $35 \mathrm{~mm} \mathrm{f} / 1.4$ lens (Rokinon, New York, NY) was mounted on the CCD camera, and a computer-controlled filter wheel was installed in front of the lens for multispectral imaging, containing four $10 \mathrm{~nm}$ band-pass filters (50 $\mathrm{mm}$ diameter; Andover Corporation, Salem, NH) spaced every 20 $\mathrm{nm}$ from 590 to $650 \mathrm{~nm}$.

The off-line system (Fig. 1B and E) is an integrated CBCT/BLT system and was previously described in (21). For CBCT imaging, similar to the SARRP setting, the animal stage was rotated between a fixed horizontal setup of an X-ray source and a detector panel. The X rays were provided by a micro-focus $\mathrm{X}$-ray source (maximum voltage $130 \mathrm{kVp}$ and maximum current $0.5 \mathrm{~mA}$; Thermo Fisher Scientific, Waltham, MA) which can automatically tune the spot size from 10 to $100 \mu \mathrm{m}$ depending on the applied voltage and current. A $15 \mathrm{~cm} \times 12$ $\mathrm{cm}$ CMOS panel (PerkinElmer) with $75 \mu \mathrm{m}$ pixel size was employed as the detector panel. The CBCT settings used in this study is $65 \mathrm{kVp}$ and $0.45 \mathrm{~mA}$ with the spot size about $41 \mu \mathrm{m}$. The smaller X-ray spot size mainly determined the higher spatial resolution in the off-line CBCT than that of the SARRP. The CBCT volume was reconstructed at a $486 \times 384 \times 256$ matrix with a voxel size of $0.3 \times 0.3 \times 0.3 \mathrm{~mm}^{3}$. For BLT, similar to the on-line system, a 3-mirror system, computercontrolled filter wheel and CCD camera mounted with a Rokinon 35 $\mathrm{mm} \mathrm{f} / 1.4$ lens were aligned perpendicular to the $\mathrm{X}$-ray imaging axis. The CCD camera, lens, filter wheel and filters were the same as those chosen for the on-line BLT system. The imaging parameters for the multispectral bioluminescence images were also consistent with the setting for the on-line system. 
A On-line SARRP BLT system

B Off-line BLT/CBCT system
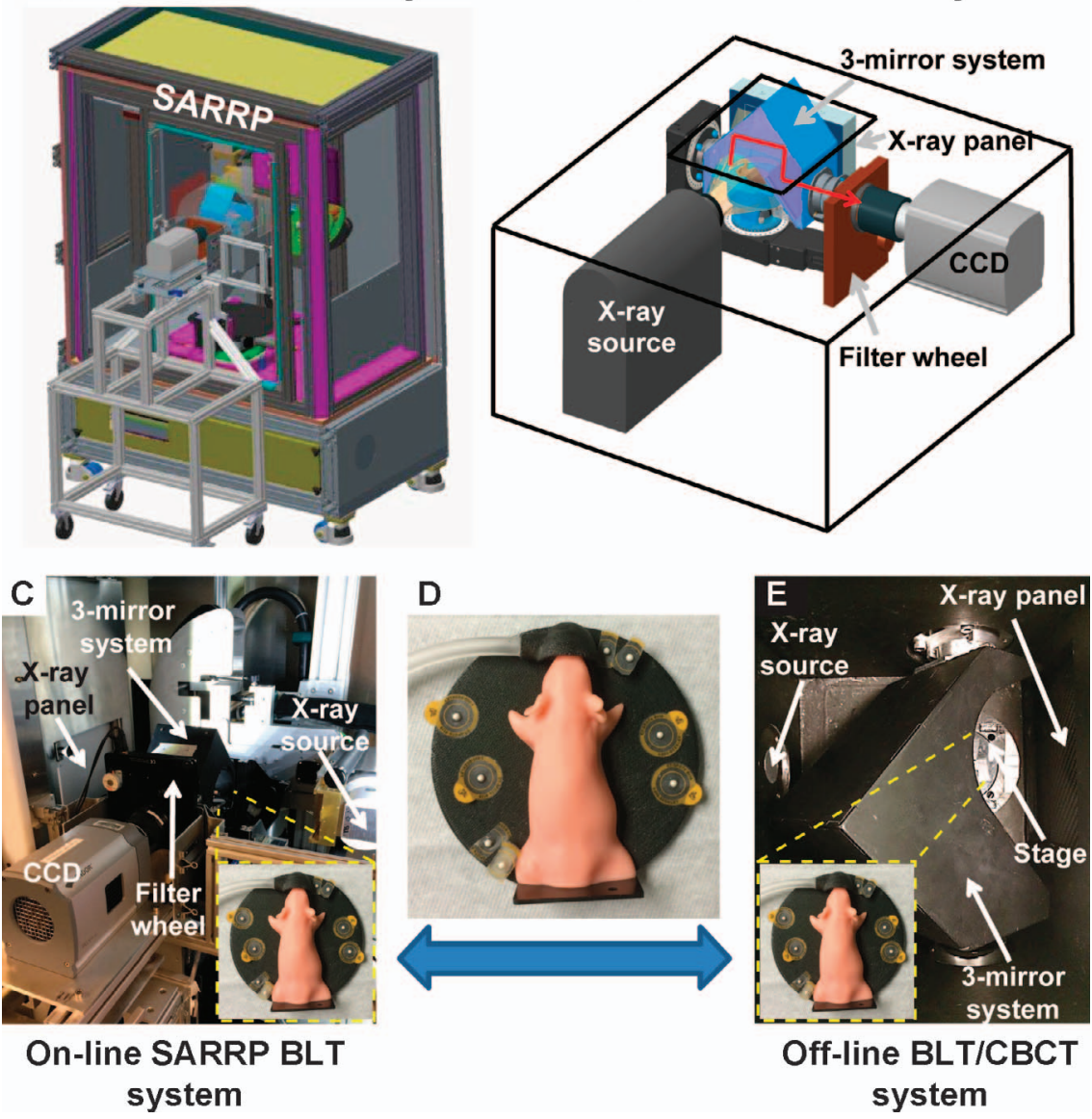

FIG. 1. Panels A and B: Drawings of the on-line SARRP BLT and off-line BLT/CBCT systems, respectively. Both systems adopt the similar configuration of the CBCT and optical assembly. The optics assembly contains the 3-mirror system, filter wheel and CCD camera. CBCT is acquired with X-ray source and detector panel in the horizontal setup. The 3-mirror system reflects the bioluminescence emitted from the mouse surface to the CCD camera via the optical path (red arrow). For the off-line system, all the components are enclosed in lead shielding. Panel C: Actual on-line SARRP BLT system. Panel E: Imaging chamber of the off-line system. Panel D: Portable mouse bed used for animal transport between the on-line and off-line systems. A gas tube was connected to the isoflurane/oxygen flow to keep the mouse anesthetized during transport and imaging procedures. In accordance with NIH requirements, a mouse phantom instead of a real animal is shown (panels C and $\mathrm{E}$ inserts, panel D) to illustrate the animal being imaged and transported between the two systems. In the real animal experiments, the mouse was taped for immobilization and eight lead pellets were used as imaging markers.

\section{Animal Preparation and Source Implantation}

Five mice $(\mathrm{FVB} / \mathrm{N}, \sim 20$ weeks old) were used in the following CBCT and off-line BLT study in accordance with the guidelines from the Johns Hopkins Animal Care and Use Committee. After the mouse was anesthetized with $1-2 \%$ isoflurane in oxygen, the hair was shaved in the abdomen area, followed by chemical hair depilation (Nair; Church \& Dwight Co., Lakewood, NJ). With the mouse in supine position, the abdomen was prepped with iodine (10\% topical solution) and draped appropriately. A transversal incision was made in the abdomen, the colon was gently lifted and a self-illuminated source $\left(0.9 \mathrm{~mm}\right.$ diameter $\times 2 \mathrm{~mm}$ length, trigalight ${ }^{\circledR} ; \mathrm{mb}$-microtec ag, Niederwangen, Switzerland) was placed in the abdomen. The peritoneum and muscle of the incision were sutured with nylon $7 / 0$ (AROSurgical $^{\mathrm{TM}}$ Instruments Inc., Newport Beach, CA), followed by suturing of the skin with nylon 6/0. After the incision, the mouse was taped on the animal stage. The depth of the implanted source was 3-5 $\mathrm{mm}$. The entire surgical procedure lasted approximately 15 min per mouse. After the experiments, the mice were euthanized.

\section{Animal Transport and CBCT Analysis}

To assess the setup accuracy during animal transport in between the SARRP and off-line system, the mice underwent CBCT imaging on the SARRP and were subsequently transported to the off-line system (Fig. 1) for a second CBCT imaging session. These two CBCT images were compared to evaluate if the transport process perturbed the animal positioning. The distance between the two systems is within 5 m. BLT-guided irradiation was facilitated via a transport bed where the animal remained anesthetized and stationary. Figure 1D shows the detachable animal bed, which supports isoflurane/oxygen delivery, is equipped with imaging markers and can be fitted on the SARRP for imaging and irradiation. In accordance with published requirements by the National Institutes of Health $(\mathrm{NIH})$, a mouse phantom instead of real animal is shown in Fig. 1D. During the experiment, the mouse was taped to the bed in a supine position. The gas tube connected to the portable bed can be switched on and off and reconnected to the anesthesia machine at each system after transport. Because the orientations of the CBCT coordinate systems are different between the 
two systems, eight fiducial markers (CT-Spot, $2.3 \mathrm{~mm}$ diameter; Beekley Medical ${ }^{\circledR}$, Bristol, CT) were placed on the bed and used to register the CBCT coordinate systems. A MATLAB ${ }^{\circledR}$ function, imregtform (MathWorks ${ }^{\circledR}$ Inc., Natick, MA), was used to register the two CBCT coordinate systems through the transformation matrix established from the fiducial markers shown in both images. Because the registration was established in the voxel space, the similarity transform method from the imregtform was chosen to scale the images at equal voxel space, and translation and rotation shift between the two CBCT coordinate systems were calculated. Of note, the registration was solely based on the marker positions but not on the animal image, to account for the spatial deviation of the two CBCT coordinate systems. Analysis of the CBCT images was confined to the mouse torso. The images were background subtracted and normalized to the maximum intensity, and background intensity was set to 0 based on a threshold level. Histogram of the intensity difference and the correlation of the two CBCT volumes were analyzed to evaluate the perturbation of the animal positioning during the transport. The correlation was quantified by plotting the normalized CBCT image intensity of the off-line system versus that of the SARRP for a given image voxel position. A strong linear relationship is expected if the two images are correlated. To further examine the sensitivity of the histogram and the correlation analysis to the animal positioning, we intentionally shifted the off-line CBCT image in $\mathrm{x}$-axis (from animal right to left side) and y-axis (from head to tail) by 1,2 and $3 \mathrm{~mm}$, respectively, and compared the shifted image with the nonshifted SARRP CBCT image.

\section{Off-Line BLT Localization}

To determine if an off-line BLT system without CBCT is applicable to guide irradiation, two scenarios were devised and compared: 1. A 3D bioluminescent source reconstruction based on the on-line SARRP multispectral bioluminescence images and the SARRP CBCT; and 2. Reconstruction based on the bioluminescence images acquired on the off-line BLT system and the SARRP CBCT. The CBCT is required to generate the object mesh used in the BLT reconstruction (19). The first scenario is assumed to have the least setup error, because no animal transport is involved. For the second scenario, the animal was transported to the SARRP for CBCT imaging. In support of a standalone BLI/BLT-guided application, the second scenario was used to evaluate the accuracy of target localization for irradiation when the off-line bioluminescence system is in close proximity to the SARRP while the animal remains properly anesthetized and immobilized. The same mouse was used for the first and second scenarios. The bioluminescence images were acquired followed by the CBCT imaging. The multispectral bioluminescence images were acquired with $4 \times 4$ binning $(0.6 \mathrm{~mm} / \mathrm{pixel})$ and exposure time ranging from 60 to $200 \mathrm{~s}$ per wavelength, depending on the source position.

Before BLT reconstruction, the 2D bioluminescence images were calibrated for the intensity nonuniformity arising from the lens vignetting effect and nonuniform pixel response of the CCD chip, then mapped onto the CBCT using a 2D transformation matrix $(19,21)$. Single projection of the bioluminescence images, from mouse anterior to posterior direction, mouse at supine position, was used for this study. A section of the mouse torso was cropped from the CBCT image to generate a 3D tetrahedral mesh $(\sim 10,000$ nodes and 50,000 elements) using the open source software NIRFAST $(22,23)$ for the subsequent BLT reconstruction. The detail of the reconstruction algorithm has been previously reported elsewhere (19). Briefly, the light propagation in tissue is described by the diffusion equation and the incomplete variables truncated conjugate gradient (IVTCG) algorithm with adaptive shrinking strategy $(19,24,25)$ was chosen as the reconstruction scheme. The optical properties, as described in our previously published work, were selected to describe the light absorption and scattering in the abdomen region (19). For the absorption coefficients $\mu_{a}$, the values are $0.043,0.013,0.007$ and $0.005 \mathrm{~mm}^{-1}$ at $590,610,630$ and $650 \mathrm{~nm}$ and the corresponding reduced scattering coefficient $\mu_{s}$ ' are $1.53,1.46,1.4$ and $1.35 \mathrm{~mm}^{-1}$. The refractive index of 1.4 between tissue and air interface was used for all in vivo experiments (26). To account for the positioning uncertainty of the mesh node generated from the NIRFAST module, three independent calculations based on three meshes were performed for each BLT run. The CoM of the reconstructed source was calculated, and the deviation between the BLT reconstructed CoM and that from the CBCT was compared, where the CoM of the source on CBCT was taken as "truth". The 3D BLT reconstruction time ranges from 1 to $4 \mathrm{~min}$, depending on the number of mesh nodes and the spatial volume of the solution searching space. The calculations were performed on a 64-bit laptop with an Intel ${ }^{\circledR}$ Core $^{\mathrm{TM}}$ i7-3920XM 2.9$\mathrm{GHz}$ processor and $32 \mathrm{~GB}$ of memory.

\section{In Vivo BLT Based on the Standalone System at the University of Pennsylvania}

A BLT guidance system was constructed for Department of Radiation Oncology, Perelman School of Medicine, where specific modifications were needed to accommodate for limited space. In particular, the system operates exclusively as an off-line standalone system in close proximity to the SARRP, less than $4 \mathrm{~m}$ between the two systems. The BLT system is not equipped with CBCT modality. After the bioluminescence imaging sessions, the mouse was transported to the SARRP for CBCT imaging. The BLT reconstruction was based on the bioluminescence images acquired from the BLT system as well as the mesh of the imaged animal, generated from the SARRP CBCT. A one-dimensional (1D) linear stage was adopted to manually drive the mouse into the light enclosure (Fig. 5A). A detachable bed supports isoflurane delivery and can be fitted on the BLT system for imaging and the SARRP for irradiation. A filter wheel and a lens were mounted in front of the CCD camera, and a mirror was used to reflect light to the CCD camera (Fig. 5B). To increase BLI acquisition speed, a shorter optical path, compared to the BLT systems shown in Fig. 1 ( 25 vs. $42 \mathrm{~cm}$ ), was achieved by using a single $45^{\circ}$ mirror. The fixed $45^{\circ}$ mirror supports single-view imaging, as in the commercial PerkinElmer IVIS system (18). Except the mirror and optical path arrangements, all optical components were the same as those in the on-line and off-line systems (Fig. 1).

The phantom experiments, which have been previously described (19), were performed to validate the target localization accuracy for the system at University of Pennsylvania. The tissue-mimicking phantom $(19,27)$ embedded with a self-illuminated source (cylindrical shape, $0.9 \mathrm{~mm}$ in diameter and $2 \mathrm{~mm}$ in length) was fixed on the detachable mouse bed and imaged in the standalone BLT system at University of Pennsylvania. The phantom was later transported to the SARRP for CBCT imaging. Multispectral bioluminescence images at $590,610,630$ and $650 \mathrm{~nm}$ were acquired with $20 \mathrm{~s}$ exposure time per wavelength and $8 \times 8$ binning $(0.8 \mathrm{~mm} /$ pixel $)$. A section of the phantom was cropped from the CBCT image to generate a 3D tetrahedral mesh $(\sim 11,000$ nodes and 60,000 elements $)$ for BLT reconstruction. The relative spectral weights of the light sources were $0.92,1,0.96$ and 0.54 at wavelengths $590,610,630$ and $650 \mathrm{~nm}$, respectively. The $3 \mathrm{D}$ source distribution was reconstructed using the same refractive index and optical properties presented in our previously published work $(19,32)$, i.e., the refractive index was 1.56 , the absorption coefficients $\left(\mu_{a}\right)$ were $0.014,0.009,0.008,0.008$ $\mathrm{mm}^{-1}$ at 590, 610, 630 and $650 \mathrm{~nm}$ and the corresponding reduced scattering coefficients $\left(\mu_{s}^{\prime}\right)$ were $0.816,0.756,0.733$ and $0.725 \mathrm{~mm}^{-1}$. Three separate experiments were performed.

To demonstrate the in vivo target localization, a flank tumor model was established by subcutaneously injecting firefly luciferaseexpressing cells (pancreatic tumor cells derived from a spontaneous pancreatic tumor KPC mouse model, $\sim 2 \times 10^{5}$ cells) into a syngenetic host (C57BL/6J). The mouse was imaged approximately one month after cell injection. A dose of $200 \mu \mathrm{l}$ luciferin $(15 \mathrm{mg} / \mathrm{ml})$ was administered by intraperitoneal injection before imaging. Multispectral bioluminescence images at 590,610,630 and 650 

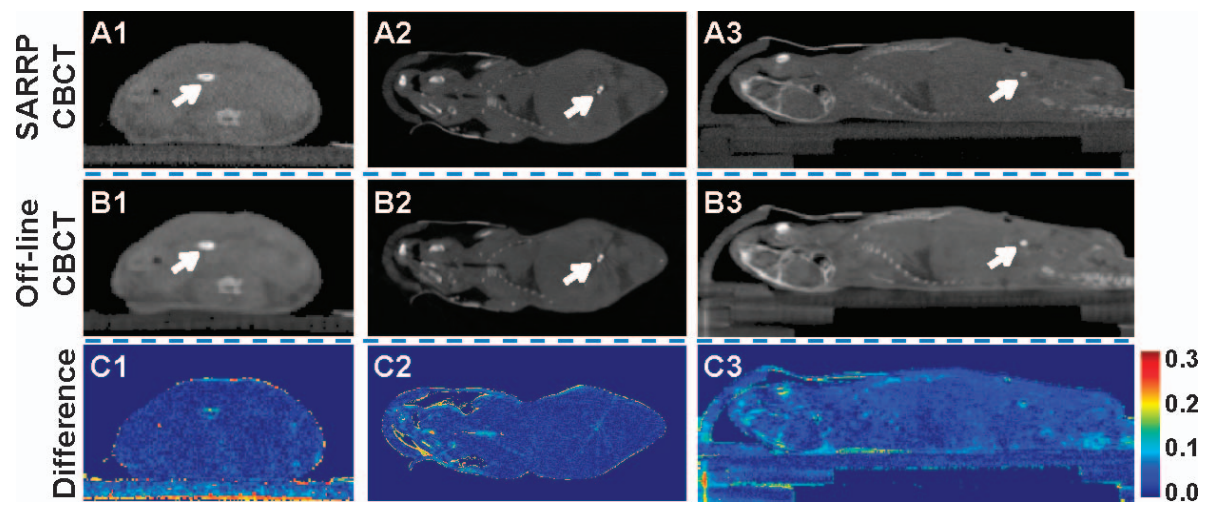

FIG. 2. Panels A1-3: Transverse, coronal and sagittal views of the normalized SARRP CBCT. Panels B1-3: The corresponding CBCT images were acquired from the off-line system. The arrow indicates the position of the embedded self-illuminated source. Panels C1-3: Intensity difference between panels A1-3 and B1-3.

$\mathrm{nm}$ were acquired with $120 \mathrm{~s}$ exposure time per wavelength and $8 \times$ 8 binning $(0.8 \mathrm{~mm} / \mathrm{pixel})$. After bioluminescence image acquisition the animal, immobilized in a detachable mouse bed, was transferred to the SARRP for CBCT imaging. A section of the torso was cropped from the CBCT image to generate a 3D tetrahedral mesh $(\sim 10,000$ nodes and 51,000 elements) for BLT reconstruction. The relative spectral weights of the luciferase-expressed cells were measured by imaging the cells in petri dishes $\left(30 \mathrm{~mm}\right.$ in diameter, $\sim 5 \times 10^{5}$ cells/ dish, $50 \mu \mathrm{l}$ luciferin $(15 \mathrm{mg} / \mathrm{ml}) / \mathrm{dish})$. The open field images (without filter) were also acquired before, in between and after the bioluminescence imaging to quantify the in vitro signal variation over time. The imaging time of each image was recorded and the curve describing the in vitro time-resolved signal variation was generated. To eliminate the influence of in vitro bioluminescent signal changing over time, the intensity of the bioluminescence image at four wavelengths taken at different time points was scaled, based on the time-resolved curve. In this study, six dishes were imaged and the average relative spectral weights were $1,0.56,0.35$ and 0.13 at $590,610,630$ and $650 \mathrm{~nm}$, respectively. The timeresolved in vivo bioluminescent signal variation was quantified using a similar approach to compensate the signal changing during the multispectral bioluminescence image acquisition. The time-resolved in vivo bioluminescent signal variation factors were $0.92,0.99,1$ and 0.86 at the 590, 610, 630 and $650 \mathrm{~nm}$, respectively, and the acquired image at each wavelength was divided by the corresponding factor. The optical properties for the subcutaneous tumor were used (28), i.e., for the absorption coefficients $\mu_{a}$, the values were $0.38,0.23$, 0.19 and $0.16 \mathrm{~mm}^{-1}$ at $590,610,630$ and $650 \mathrm{~nm}$, respectively, and the corresponding reduced scattering coefficients $\mu_{s}$ ' were $0.9,0.76$, 0.69 and $0.66 \mathrm{~mm}^{-1}$. The BLT reconstruction algorithm described above was applied for the subcutaneous model reconstruction.

\section{RESULTS}

\section{Animal Transport and Positioning Accuracy}

The mouse CBCT was acquired on both the SARRP and off-line system by transferring the animal between systems over a distance of less than $5 \mathrm{~m}$. Figure 2 shows a representative comparison between the mouse CBCT acquired from the SARRP (Fig. 2A1-A3), and the images acquired from the off-line system (Fig. 2B1-B3). The arrows indicate the location of the implanted source. The deviation of the implanted source center between the two systems was accurate at $0.1 \pm 0.03 \mathrm{~mm}$. One would expect the region with strong intensity difference between the two images to represent a large positioning deviation that occurred during the transport. The larger difference can be observed at the mouse surface, and minimal differences are observed in the internal structures (Fig. 2C1-C3). With the anesthetized animal properly immobilized, the change in the mouse position and posture due to transport was minimal.

The corresponding histogram of the intensity difference showed quantitatively that minimal deviation of the animal positioning resulted from animal transport between systems (Fig. 3A1). The histogram fitted by a Gaussian distribution shows the full width at half maximum (FWHM) as 0.07 and the 3 standard deviations at -0.08 and 0.09 . The correlation between the two CBCT volumes was quantified in the Fig. $3 \mathrm{~A} 2$. A strong linear relationship (correlation coefficient $=$ 0.99) is shown between the normalized off-line CBCT intensity and that of the SARRP system. The discontinuities in the low-intensity area (Fig. 3A2) resulted from the pixels at the image background area that were not entered into the study. The histogram and correlation analysis corresponding to the $2 \mathrm{~mm}$ shift applied to the off-line CBCT image in $\mathrm{x}$ axis (no shift applied to the SARRP CBCT) are shown in Figs. 3B1 and B2, respectively. Although Gaussian distribution is not the best fit to describe the histogram with image shift (Fig. 3B1), qualitatively, the spread of the Gaussian fit is broader, (FWHM $=0.34$ and the 3 standard deviations are at -0.42 and 0.43 ) compared to the histogram without shift (Fig. 3A1 versus 3B1). Interestingly, there are two small humps close to the extreme ends of the histogram in Fig. 3B1 and several points also appear on the two axes in the correlation analysis in Fig. 3B2, which were caused by the intensity difference at the mouse boundary due to the shift. As expected, the correlation between the two CBCT volumes with the $2 \mathrm{~mm}$ positioning deviation is weak (correlation coefficient $=0.68$ ). The FWHM from the Gaussian fit to the histogram and the correlation coefficient for different amounts of shifts in both the $\mathrm{x}$ - and $\mathrm{y}$-axes are shown in Fig. $3 \mathrm{C} 1$ and $3 \mathrm{C} 2$. The average and standard deviation from all five mice are shown in Fig. $3 \mathrm{C} 1$ and $3 \mathrm{C} 2$. 

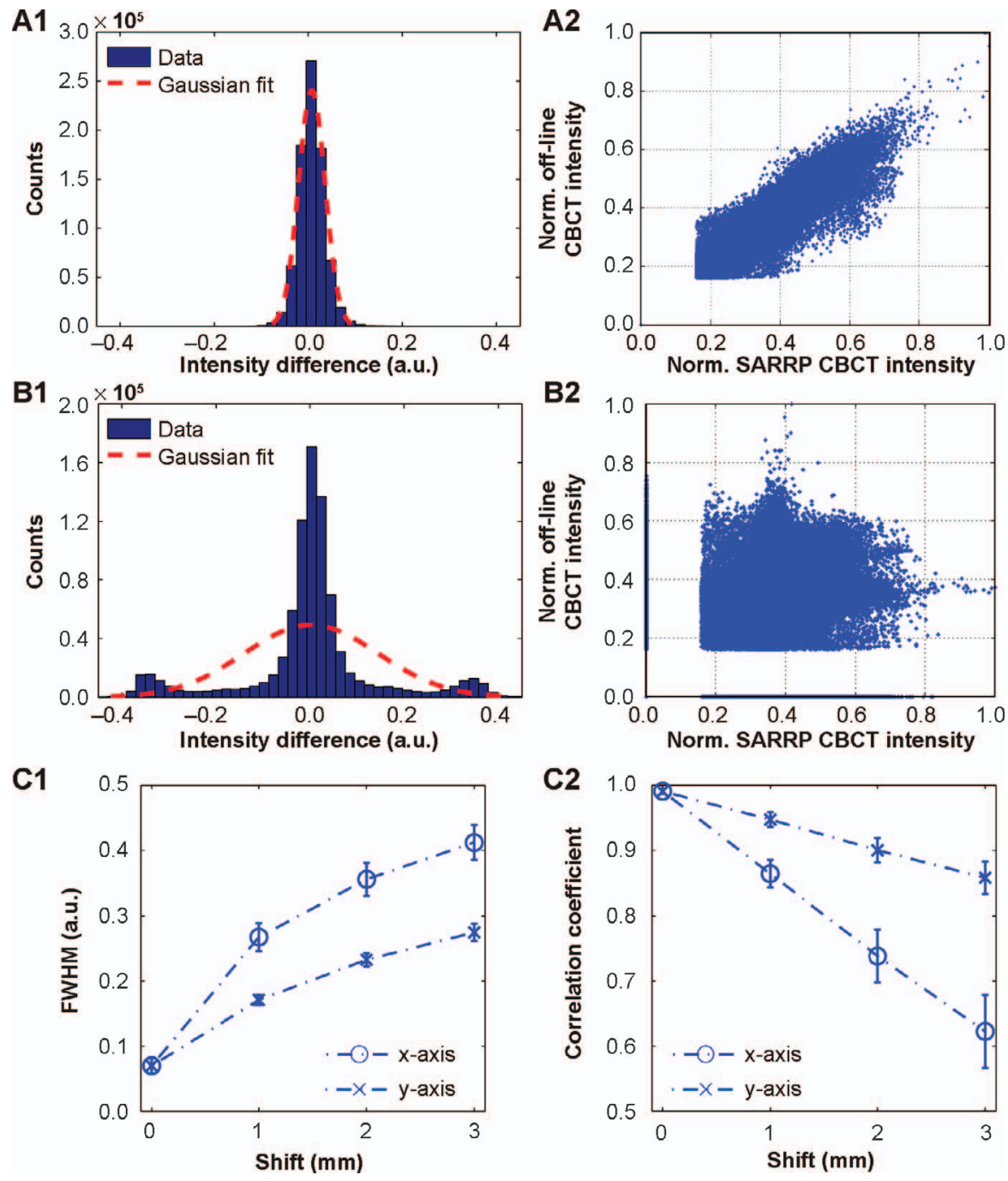

FIG. 3. Panel A1: Representative histogram of the intensity difference between the SARRP and the off-line CBCT with Gaussian fit. Panel A2: Corresponding correlation analysis between the normalized off-line CBCT intensity and the SARRP system for a given image voxel position. The two data sets are strongly correlated (correlation coefficient $=0.99$ ). Panels B1 and B2: Corresponding histogram and correlation analysis, respectively, while the $2 \mathrm{~mm}$ shift along the $\mathrm{x}$-axis was applied to the off-line CBCT image. Panel B2: The two data sets show a weak correlation (correlation coefficient $=0.68$ ). Panel C1: Average FWHM with standard deviation (5 mice) of the Gaussian fit for the intensity difference histograms for various shifts applied to the offline CBCT image in $\mathrm{x}$ - and $\mathrm{y}$-axis, while the SARRP CBCT images were fixed. Panel C2: Corresponding correlation coefficients with standard deviation. The error bars for 0 shift in panels $\mathrm{C} 1$ and $\mathrm{C} 2$ are smaller than the symbols.

As the amount of shifts is increased, the FWHM will be larger and the correlation weaker. Fig. $3 \mathrm{C} 1$ and $3 \mathrm{C} 2$ also show what the expected FWHM and the correlation coefficient should be with minimum positioning difference.

\section{Off-Line BLT Localization}

To assess if the off-line BLT system is applicable to guide irradiation, we compared 3D bioluminescent source reconstruction based on the on-line SARRP multispectral bioluminescence images and the SARRP CBCT (Fig. 4A1-A3) with the reconstruction based on the off-line bioluminescence images and the SARRP CBCT (Fig. 4B1B3). The geometric coordinates of the SARRP CBCT and off-line BLT system were registered. As shown for both cases, we observed a close congruence between the reconstructed and true source location. The second scenario (Fig. 4B1-B3) further showed that accurate target localization in a live animal can be maintained with off-line BLT in close proximity to the SARRP. From five independent experiments, our results showed the reconstructed CoM of the trigalight source has an offset of $1.0 \pm 0.4 \mathrm{~mm}$ from the "true" CoM of the SARRP CBCT. The results are comparable with the offset of $1.0 \pm 0.2 \mathrm{~mm}$ using on-line BLT. These findings support the utilization of an off-line BLT system for accurate low-contrast soft-tissue target localization. 

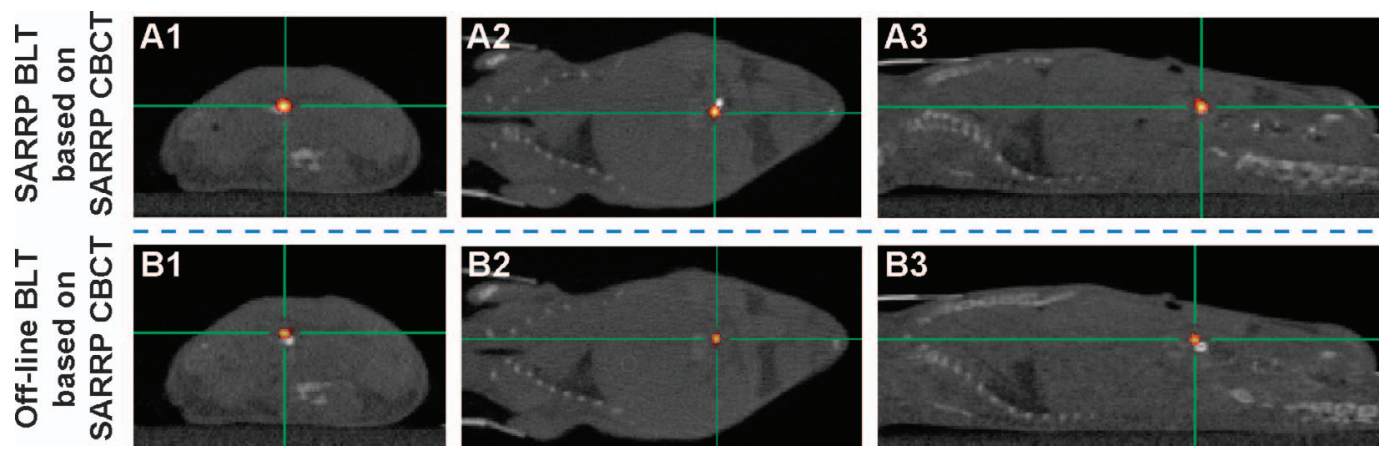

FIG. 4. A cylindrical self-illuminated light source $(0.9 \mathrm{~mm}$ diameter $\times 2 \mathrm{~mm}$ length, white dots shown in CBCT) was implanted in the mouse abdomen. Panels A1-3: Overlay of BLT with CBCT at the transverse, coronal and sagittal view, respectively. The source was reconstructed based on the on-line SARRP bioluminescence image and SARRP CBCT. Panels B1-3: Off-line BLT reconstruction based on the SARRP CBCT. The cross is centered at CoM of the reconstructed source. Voxels with reconstructed source strength greater than $10 \%$ of the maximum value among all the voxels are shown.

\section{A Standalone BLT-Guided System at University of Pennsylvania}

A photograph and schematic of the standalone BLT system at University of Pennsylvania are shown in Fig. 5A and $\mathrm{B}$. The phantom studies demonstrated that the average $3 \mathrm{D}$ offset between the BLT reconstructed CoM and the true source center is $0.8 \pm 0.02 \mathrm{~mm}$. The same phantom experiment was also performed in the off-line BLT and on- line SARRP BLT system (19), which also shows approximately $1 \mathrm{~mm}$ localization accuracy. Except for the shorter optical path and single mirror chosen for the BLT system at University of Pennsylvania, there is no difference in terms of hardware and software configuration between the University of Pennsylvania system and the off-line BLT system (compare Fig. 5A with Fig. 1B and E). Based on single projection, we expect the same localization accuracy
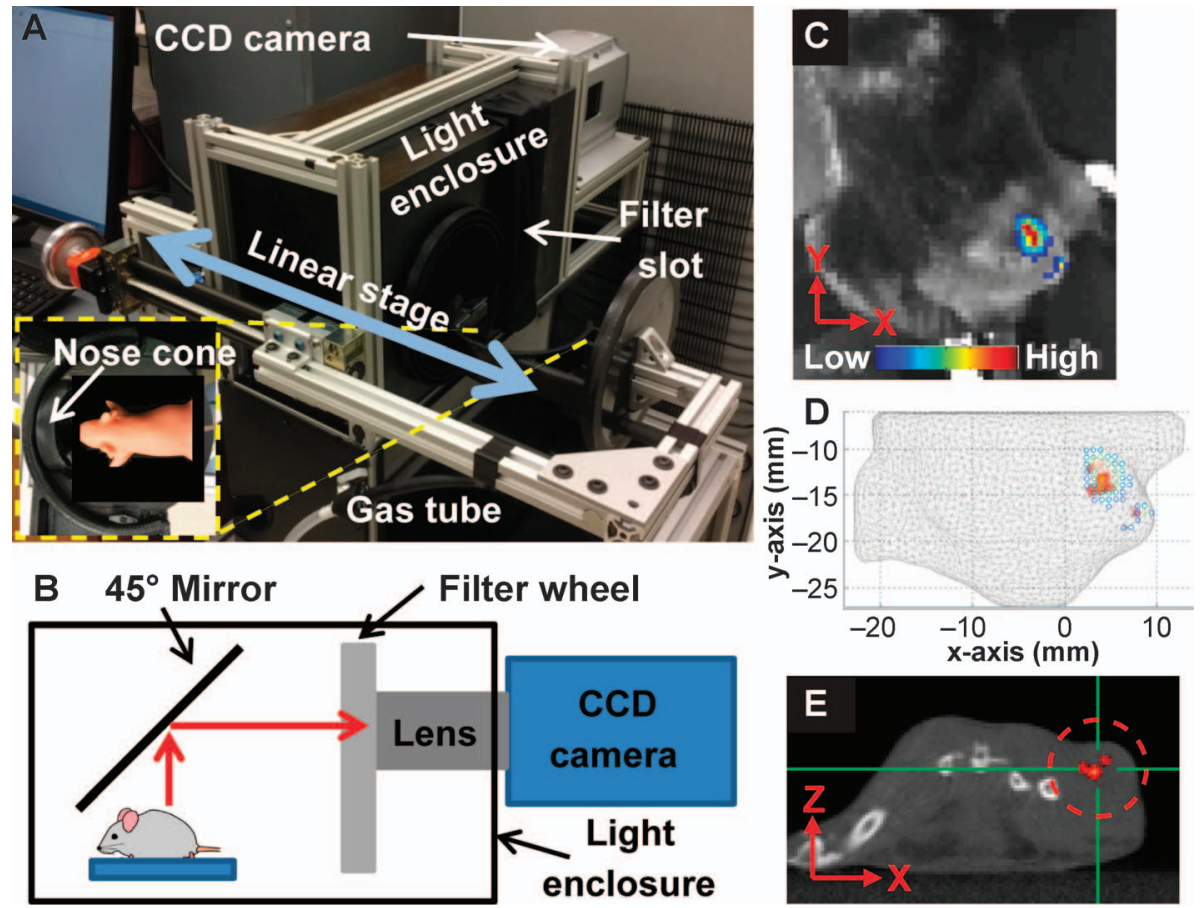

FIG. 5. Panel A: Illustration of the standalone BLT system at University of Pennsylvania Radiation Oncology Department. The detachable mouse bed is moved by a 1D manual-drive linear stage into the light enclosure. The gas tube linked to the nose cone is connected to the anesthesia machine to keep the mouse anesthetized during the imaging, transport and irradiation processes. Panel B: A filter wheel and lens were mounted in front of the $\mathrm{CCD}$ camera, and the signal is reflected to the $\mathrm{CCD}$ camera through a $45^{\circ}$ mirror. Panel $\mathrm{C}$ : Bioluminescence image from a flank tumor imaged with the system. Panel D: Bioluminescence lies on top of BLT reconstructed light source (red dots) in a 3D mesh. Panel E: The BLT reconstructed source, with the crosshair depicting its CoM, lies inside the tumor volume on CBCT. 
of the University of Pennsylvania system compared to that of the off-line BLT system. A subcutaneous model with firefly luciferase-expressing pancreatic tumor cells was imaged with the system (Fig. 5C) to demonstrate the in vivo localization capability. Figure 5D shows the reconstructed 3D distribution lying beneath the bioluminescence intensity on the surface, as expected for a subcutaneous target at shallow depth. Figure 5E shows that the reconstructed bioluminescence CoM is also within the tumor as depicted by CBCT. Our efforts are ongoing to utilize an orthotopic model for in vivo BLT validation.

\section{DISCUSSION}

In preclinical radiation research, CT/CBCT has limitations when localizing soft-tissue targets, especially for small, nonpalpable, orthotopic or spontaneous tumor models. BLI has emerged as a noninvasive means for in vivo imaging and has been applied to guide irradiation and assess tumor response in radiation therapy $(10,20,29-31)$. As we previously reported $(19,32), 2 \mathrm{D}$ BLI is not sufficient to guide irradiation. The surface light intensity is a function of the optical path from the bioluminescent targets and the maximum surface intensity does not necessarily point to the center of the internal source. A vertical beam directed to the highest bioluminescence intensity on the animal surface deviates from the CoM of an internal source by at least 3 $\mathrm{mm}$ or more (10). Including other sources of setup uncertainty (11), an undesirably large beam aperture $>15$ $\mathrm{mm}$ in diameter would be required to ensure coverage of a small $3 \mathrm{~mm}$ diameter tumor. To accurately localize orthotopic targets and utilize the advanced focal irradiation through multiple gantry angles, similar to human treatment, knowledge of the target position in 3D is required. BLT emerges as an attractive solution to guide the focal irradiation of low contrast soft-tissue targets $(19,32)$.

Tuli et al. (20) investigated the accuracy of off-line BLT to localize soft-tissue targets, by implanting a small glass bulb containing bioluminescent cells in the abdomen of a mouse carcass. The 3D deviation of the respective source centroids between BLT and CBCT was $1.5 \pm 0.9 \mathrm{~mm}$. From the Tuli et al. study (20), at least 15 min were needed to transport the animal between the systems located at different facilities. Given the rigidity of the carcasses, these results likely represent the best that can be achieved with the off-line BLT systems, which are not in close proximity to the irradiators. The setup error introduced during the animal transport and the throughput are the major obstacles to implementing this procedure for practical use. With the online BLT SARRP system (Fig. 1A and C), we can achieve target localization accuracy at $1 \mathrm{~mm}$ using an implanted source in live mice (Fig. 4A1-A3). The 3D BLT system can significantly reduce the margin to $2.5 \mathrm{~mm}(11)$ and the radiation collimator size to $8 \mathrm{~mm}$ to cover a 3-mm-diameter target, superior to 2D BLI targeting. Although the on-line system represents the most ideal case for BLT-guided application due to minimum setup error, it requires manual docking, which can be labor intensive. While one may consider permanently mounting an optical system onto the SARRP, it is no small task to add such a system to a small animal irradiator. The potential obstacles are cost, labor, lower throughput and technical challenges to modifying the existing system. Many researchers use BLI/BLT to monitor tumor growth longitudinally after irradiation without use of the SARRP, thereby freeing up the SARRP itself for irradiation time. Permanently mounting an optical system to the irradiator would limit the use of both systems. An alternative is an off-line BLT system in close proximity to the SARRP. This setting has the advantages of parallel operation for both BLI/BLT and irradiation, and provides an attractive solution for institutes with existing animal irradiators.

We evaluated the accuracy of target localization for irradiation when the off-line imaging system is in close proximity to the SARRP, while the animal remains properly anesthetized and immobilized. Analysis of the two sets of CBCT, acquired using both SARRP and off-line systems, demonstrates that minimal change in the mouse position occurred due to transport (Figs. 2 and 3). In addition, the deviation of the light source center between the two systems is less than $0.2 \mathrm{~mm}$. These results demonstrate that the setup error and organ deformation during the transport are minimal if the systems are in close proximity. The results shown in Fig. 4 further demonstrate that off-line BLT can be used to accurately localize a bioluminescent target in a live animal. The CoM of the trigalight reconstructed using off-line BLT has an average accuracy of $1.0 \mathrm{~mm}$, equivalent to the localization accuracy provided by the on-line BLT. These findings led us to develop the standalone BLT system at University of Pennsylvania (Fig. 5), which can perform soft-tissue image guidance for irradiation and can operate as an independent optical apparatus for longitudinal studies. For the lung region, the method of implanting a light source may be less ideal. A single lung nodule model (33) could be adopted for the purpose of BLT validation, since the target can be clearly localized in a CBCT image.

We note, however, that the reduction in efficiency is compounded by the need to carefully maintain anesthetization during animal transport over a short distance. From this aspect, the on-line approach remains desirable. We therefore envision a dual-use optical system (34) where a novel docking arrangement allows convenient access to CBCT on the SARRP without disrupting the animal. The dual-use system, which is motorized, can dock onto an independent mouse-supporting bed for longitudinal BLI/BLT application or target localization; it can also be driven into the SARRP for on-line irradiation guidance and assessing treatment response if a rapid radiobiological mechanism is expected. It provides significant efficiency over the manual docking system (Fig. 1A). With the capability to support off-line longitudinal studies independent of the SARRP, the dual- 
use system is an efficient and cost-effective platform to facilitate optical imaging for preclinical radiation research.

\section{CONCLUSION}

In summary, this study demonstrates that when an off-line BLT system is in close proximity to the SARRP and the study animal is effectively immobilized during transport, accurate target localization can be achieved, comparable to the on-line system, to guide SARRP irradiation.

\section{ACKNOWLEDGMENTS}

The authors thank Esteban Velarde for SARRP assistance. This work was supported by the National Institutes of Health (grant no. R01 CA 158100) and Xstrahl Ltd. (no. 90043185). Drs. Wong and Iordachita receive royalty payment from a licensing agreement between Xstrahl Ltd. and Johns Hopkins University.

Received: February 18, 2016; accepted: August 25, 2016; published online: November 21, 2016

\section{REFERENCES}

1. Clarkson R, Lindsay PE, Ansell S, Wilson G, Jelveh S, Hill RP, et al. Characterization of image quality and image-guidance performance of a preclinical microirradiator. Med Phys 2011; 38:84556.

2. Jensen MD, Hrinivich WT, Jung JA, Holdsworth DW, Drangova $\mathrm{M}$, Chen $\mathrm{J}$, et al. Implementation and commissioning of an integrated micro-CTRT system with computerized independent jaw collimation. Med Phys 2013; 40:081706.

3. Song KH, Pidikiti R, Stojadinovic S, Speiser M, Seliounine S, Saha D, et al. An x-ray image guidance system for small animal stereotactic irradiation. Phys Med Biol 2010; 55:7345-62.

4. Wong J, Armour E, Kazanzides P, Iordachita I, Tryggestad E, Deng $\mathrm{H}$, et al. High-resolution, small animal radiation research platform with X-ray tomographic guidance capabilities. Int J Radiat Oncol Biol Phys 2008; 71:1591-9.

5. Zhou H, Rodriguez M, van den Haak F, Nelson G, Jogani R, Xu J, et al. Development of a micro-computed tomography-based imageguided conformal radiotherapy system for small animals. Int $\mathbf{J}$ Radiat Oncol Biol Phys 2010; 78:297-305.

6. Klerk CP, Overmeer RM, Niers TM, Versteeg HH, Richel DJ, Buckle $\mathrm{T}$, et al. Validity of bioluminescence measurements for noninvasive in vivo imaging of tumor load in small animals. Biotechniques 2007; 43:7-13, 30.

7. O'Neill K, Lyons SK, Gallagher WM, Curran KM, Byrne AT. Bioluminescent imaging: a critical tool in pre-clinical oncology research. J Pathol 2010; 220:317-27.

8. Rice BW, Cable MD, Nelson MB. In vivo imaging of lightemitting probes. J Biomed Opt 2001; 6:432-40.

9. Baumann BC, Benci JL, Santoiemma PP, Chandrasekaran S, Hollander $\mathrm{AB}$, Kao GD, et al. An integrated method for reproducible and accurate image-guided stereotactic cranial irradiation of brain tumors using the small animal radiation research platform. Transl Oncol 2012; 5:230-7.

10. Tuli R, Surmak A, Reyes J, Hacker-Prietz A, Armour M, Leubner A, et al. Development of a novel preclinical pancreatic cancer research model: bioluminescence image-guided focal irradiation and tumor monitoring of orthotopic xenografts. Transl Oncol 2012; 5:77-84.

11. van Herk M, Remeijer P, Rasch C, Lebesque JV. The probability of correct target dosage: dose-population histograms for deriving treatment margins in radiotherapy. Int J Radiat Oncol Biol Phys 2000; 47:1121-35.
12. Gu XJ, Zhang QH, Larcom L, Jiang HB. Three-dimensional bioluminescence tomography with model-based reconstruction. Opt Express 2004; 12:3996-4000.

13. Dehghani H, Davis SC, Jiang S, Pogue BW, Paulsen KD, Patterson MS. Spectrally resolved bioluminescence optical tomography. Opt Lett 2006; 31:365-7.

14. Cong WX, Wang G, Kumar D, Liu Y, Jiang M, Wang LV, et al. Practical reconstruction method for bioluminescence tomography. Opt Express 2005; 13:6756-71.

15. Kuo C, Coquoz O, Troy TL, Xu H, Rice BW. Three-dimensional reconstruction of in vivo bioluminescent sources based on multispectral imaging. J Biomed Opt 2007; 12:024007.

16. Lu YJ, Zhang XQ, Douraghy A, Stout D, Tian J, Chan TF, et al. Source reconstruction for spectrally-resolved bioluminescence tomography with sparse a priori information. Opt Express 2009; 17:8062-80.

17. Klose AD, Beattie BJ, Dehghani H, Vider L, Le C, Ponomarev V, et al. In vivo bioluminescence tomography with a blocking-off finite-difference SP3 method and MRI/CT coregistration. Med Phys 2010; 37:329-38.

18. Behrooz A, Kuo C, Xu H, Rice B. Adaptive row-action inverse solver for fast noise-robust three-dimensional reconstructions in bioluminescence tomography: theory and dual-modality optical/ computed tomography in vivo studies. J Biomed Opt 2013; $18: 76010$

19. Zhang B, Wang KK, Yu J, Eslami S, Iordachita II, Reyes J, et al. Bioluminescence tomography guided radiation therapy for preclinical research. Int J Radiat Oncol Biol Phys 2016; 94:1144-53.

20. Tuli R, Armour M, Surmak A, Reyes J, Iordachita II, Patterson MS, et al. Accuracy of off-line bioluminescence imaging to localize targets in preclinical radiation research. Radiat Res 2013; 179:416-21.

21. Yang Y, Wang KK, Eslami S, Iordachita II, Patterson MS, Wong JW. Systematic calibration of an integrated x-ray and optical tomography system for preclinical radiation research. Med Phys 2015; 42:1710-20.

22. Dehghani H, Eames ME, Yalavarthy PK, Davis SC, Srinivasan S, Carpenter CM, et al. Near infrared optical tomography using NIRFAST: Algorithm for numerical model and image reconstruction. Commun Numer Methods Eng 2008; 25:711-32.

23. Jermyn M, Ghadyani H, Mastanduno MA, Turner W, Davis SC, Dehghani $\mathrm{H}$, et al. Fast segmentation and high-quality threedimensional volume mesh creation from medical images for diffuse optical tomography. J Biomed Opt 2013; 18:86007.

24. He X, Liang J, Wang X, Yu J, Qu X, Wang X, et al. Sparse reconstruction for quantitative bioluminescence tomography based on the incomplete variables truncated conjugate gradient method. Opt Express 2010; 18:24825-41.

25. Naser MA, Patterson MS. Bioluminescence tomography using eigenvectors expansion and iterative solution for the optimized permissible source region. Biomed Opt Express 2011; 2:3179-93.

26. Virostko J, Powers AC, Jansen ED. Validation of luminescent source reconstruction using single-view spectrally resolved bioluminescence images. Appl Opt 2007; 46:2540-7.

27. Pekar J, Patterson MS. Fabrication and characterization of phantoms with tissue-like optical properties from 500 to $700 \mathrm{~nm}$. Med Laser Appl 2010; 25:147-53.

28. Honda N, Ishii K, Terada T, Nanjo T, Awazu K. Determination of the tumor tissue optical properties during and after photodynamic therapy using inverse Monte Carlo method and double integrating sphere between 350 and $1000 \mathrm{~nm}$. J Biomed Opt 2011; 16:058003.

29. Lee CJ, Spalding AC, Ben-Josef E, Wang L, Simeone DM. In vivo bioluminescent imaging of irradiated orthotopic pancreatic cancer xenografts in nonobese diabetic-severe combined immunodeficient mice: a novel method for targeting and assaying efficacy of ionizing radiation. Transl Oncol 2010; 3:153-9. 
30. Weersink RA, Ansell S, Wang A, Wilson G, Shah D, Lindsay PE, et al. Integration of optical imaging with a small animal irradiator. Med Phys 2014; 41:102701.

31. Butterworth KT, Redmond KM, McMahon SJ, Cole AJ, Jain S, McCarthy $\mathrm{HO}$, et al. Conventional in vivo irradiation procedures are insufficient to accurately determine tumor responses to nonuniform radiation fields. Int J Radiat Biol 2015; 91:257-61.

32. Yu J, Zhang B, Iordachita II, Reyes J, Lu Z, Brock MV, et al.
Systematic study of target localization for bioluminescence tomography guided radiation therapy. Med Phys 2016; 43:2619.

33. Herter-Sprie GS, Korideck H, Christensen CL, Herter JM, Rhee K, Berbeco RI, et al. Image-guided radiotherapy platform using single nodule conditional lung cancer mouse models. Nat Commun 2014; 5:5870.

34. Li M, He X, Eslami S, Wang KK, Zhang B, Wong J, et al. A dualuse imaging system for pre-clinical small animal radiation research. Conf Proc IEEE Eng Med Biol Soc 2015; 2015:6904-7. 